\title{
Review on Electrical Motor and Pump Assemblies in Ventricular Assist Devices \& Total Artificial Hearts
}

\author{
R. Basanth, Anil K. Puppala \\ ${ }^{1,2}$ Dept of Electrical \& Electronics Engineering, SNIST, Sreenidhi Institute of Science and Technology, \\ Hyderabad, India \\ Email:basanth246@gmail.com,panilkumar@sreenidhi.edu.in
}

Received: 20 ${ }^{\text {th }}$ December 2017, Accepted: 20th January 2018, Published: 28th February 2018

\begin{abstract}
Pump and motor assembly plays a vital role in the design of the Ventricular Assist Devices (VAD) \& Total Artificial Hearts (TAH). Heart's pulsatile blood flow was first mimicked by pulsatile flow devices. However, due to the compact sizing of the continuous flow devices, have shown significant decrease in the mortality rate. Pulsatile and continuous flow devices differ in their motors and drive systems. This paper presents a review of different motor structures that were used in both the systems.
\end{abstract}

Keywords-Total Artifical Heart; Ventricular Assist Devices, Pump, PMSM, BLDC motor, Linear motor.

\section{Introduction}

HEART disease is one of the major cause of death. Because of less availability of donor hearts, developments and advancements in mechanical circulatory system (Ventricular Assist Devices VADs, Total Artificial Hearts -TAHs) is going on from past 50 to 60 years[1][2]. Importance of Mechanical Circulatory System (MCS) has increased to enhance or replace myocardial performance. A mile stone in artificial organ development arose in 1943 [3]. The quest for an artificial heart started in 1957. The field of (MCS) was first introduced in 1964 [4][5]. The Patients receiving a mechanical circulatory support device (MCSD) place their lives in the hands of the engineers who design the MCSDs and the physicians who manage the MCSDs [6]. Due to shortages in donor organ availability, advanced heart-failure patients are at high risk often leading to death while awaiting transplantation, this motivates the engineers to develop MCSDs. Statistics on mortaility because of heart diseases is shown in Table 1, indicates that there're will be a $127 \%$ and $115 \%$ increase in the mortaility in India in Men and women respectively.[7]

The primary or initially artificial hearts were a great extent mechanical devices, these devices were extremely unwieldy, electrical power is given to implantable devices through outer pneumatic cables. These are introduced with pulsatile flow innovation. These devices are problematic as little exhaustion splits which forms in bladder walls, finally they leads to premature death of patient [8]. The pumps lifetime with this type of technology is about one to

two years only. By considering these issues, the biomedical group investigated for elective approach, and the new designs are in minimized structure and mechanically reliable design, as opposed to depending on to a largely mechanical devices with their characteristic weakness to weariness disappointments.

\begin{tabular}{|c|c|c|c|c|c|c|}
\hline \multirow[b]{2}{*}{ Region } & \multicolumn{3}{|c|}{ Women } & \multicolumn{3}{|c|}{ Men } \\
\hline & 1990 & 2020 & 8 Increase & 1990 & 2020 & Sinctease \\
\hline EME & 838 & 1107 & 32 & 329 & 1209 & 46 \\
\hline FSE & 559 & 702 & 26 & 458 & 712 & 52 \\
\hline Total developed countries & $139 ?$ & 1809 & 29 & 1297 & 1921 & 48 \\
\hline India & 556 & 1197 & 115 & 619 & 1406 & 127 \\
\hline China & 377 & 684 & 81 & 306 & 811 & 110 \\
\hline $\mathrm{OAN}$ & 227 & 552 & 143 & 233 & 581 & 149 \\
\hline $55 \mathrm{~A}$ & 117 & 263 & 125 & 92 & 222 & 141 \\
\hline Latin america & 169 & 412 & 14 & 179 & 444 & 148 \\
\hline Niddle East & 291 & 717 & 145 & 319 & 874 & 174 \\
\hline Total developing countries & 1737 & 3825 & 120 & 1828 & 4337 & 137 \\
\hline world & 3134 & 5634 & 130 & 3125 & 6258 & 100 \\
\hline
\end{tabular}

Table. 1. Statistics on heart diseases projected between 1990 and 2020.

Most solutions involved in rotating pump designs: either centrifugal pumps, or axial flow pumps [9]. Advancement from pulsatile flow to persistent or continuous flow designed devices has significantly enhanced as a result of very compact structure [10].

\section{Development}

MCDs had seen three phases are advancement. The intial eras of ventricular assist devices (VADs) were pulsatile devices. This flow was achieved by pusherplates. But life of those devices were very less, it is about to 2 to 3 years only. The second era of VADs utilized constant flow rotating pumps with mechanical bearings and it is reported that they seals in contact with blood[11]. Revolving blood pumps (RBPs) helps to pump blood from ventricle to aorta, mostly by-passing the aortic valve (AV) [12]. Durability of the devices increases with lessened 
mechanical versatile elements[13]. The structural sizes of second era devices are very small with initial devices. At last, the third and most recent era of VADs utilizes attractive and hydrodynamic bearing. By expelling the requirement for contact bearing framework, life of most recent era devices are about to 10 years [14]. Magnetic levitation technique is introduced in recent advancements of artificial hearts, this is a workable suspension technique, which drives the impeller of an VAD or TAH to reduce blood damage and to improve device life time also [15]. Continuous flow LVADs are simpler in structure, because of all LVADs implanted in the year of 2012 and 2013 were continuous-flow LVADs only[16]. For the treatment of end stage heart problems the rotary pump based continuous flow LVADs were introduced in recent era.
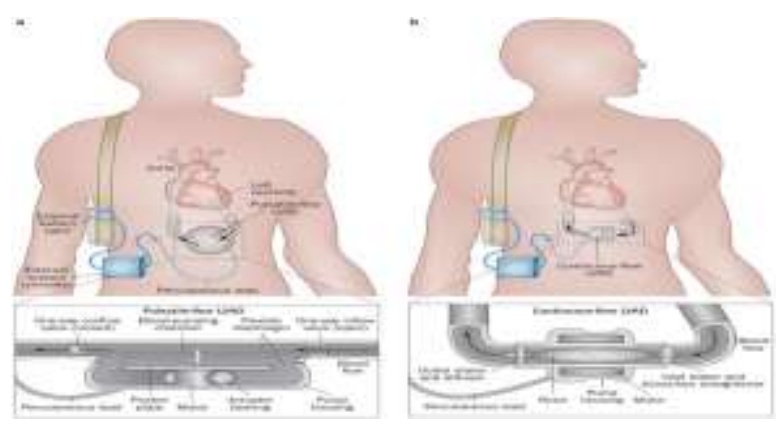

Figure. 1. (a) Pulsatile flow LVAD. (b) Continuous flow LVAD.

DIFFERENT MOTORS WERE USED IN ARTIFICIAL HEARTS AND RECENT ADVANCEMENTS.

Following are different motors that were used in TAHs and VADs.

\section{A. Linear motors :}

Rotary motors with their pumps consisted of many moving components. The reduction in wear prone parts leads to increase in the life expectancy, and smaller sizes. Linear motor drives are less wear prone parts, and were preferred for CF pumps [17]. Linear motor type drive was used in initial designs of artificial hearts and which had only one moving part. A crucial challenge in MCDs is that reduction of heat dissipation. For the purpose of LVADs and TAHs, heat loss higher than $20 \mathrm{~W}$ is not endured by the human body. essentially linear type drive topologies are either excessively weak or excessively voluminous. Based on magnetic force simulation, direct linear drives for a TAH were reported in [18] and those devices were accomplished weight, force, and size requirements [18]. Rotary pump based existing devices are[19] likewise experienced harm to blood cells. This problem had addressed in linear motor drive type artificial hearts, which was reported in [19], blood pumping capability is very poorer with linear motor used MCDs. And they also experienced reliability problems, which all are reported in [19].

\section{B. Double stator structure and a vernier topology motors :}

Double stator structure and a Vernier topology motors reported in [20-21], which are having higher force capability due to their double structure. but they suffered from a difficult manufacturability because of double structure nature and they faced low power density problems. Double structure nature increases the flux, it leads to higher force density.

\section{Tubular stator-PM veniermotor:}

Construction and advantages of Tubular stator-PM vernier motor (TSPM veniermotor) is given in [22]. Structural complexity of TSPM veiermotor is very less compared to Double stator structure and a vernier topology motor. This motor slyly coordinates ferromagnetic poles and permanant magnets together. Robust structure, higher efficiency are the advantages of TSPM veiermotor apart from high force density. Double stator tubular permanent magnet (DSTPM) motor reported in [22], which had outer and inner stators (two stators) with appropriate sizes. so cogging forces developed by outer and inner stators are counteracted by each other, therefore limiting motor cogging forces.

tubular double-stator vernier PM (TDS-VPM) motor having with higher force density, by consolidating the benefits of the double-stator structure and the vernier structure was reported in [13], The motor offered high power density and high stability. Due to double stator structure nature The TDS-VPM motor offers higher back EMF, lower cogging forces, and higher force density.

\section{Permanent magnet synchronous motor:}

Another kind of motor is that permanent magnet synchronous motor (PMSM). PMSM in [23] associated to centrifugal blood pump, PMSM is utilized to drive centrifugal blood pump. Furthermore, the essential design is that, the pump impeller is combined with shaft, where mechanical bearing provides supports to the both pump impeller and shaft of motor. But main drawback with such design is forming of blood clots on shaft and bearing due to mechanical coupling, it was reported that inefficient sealing leads in formation of blood clots on bearing and shaft of motor. And another disadvantage is it is with bulky volume. to avoid these all drawbacks in the conventional design, researchers introduced a revised artificial heart with magnetic coupler and magnetic bearing, Which is also reported in [23]. Such design overcame all the drawbacks in previously designed artificial hearts. The maximum speed reported with magnetically suspended type design about to $730 \mathrm{r} / \mathrm{min}$. 


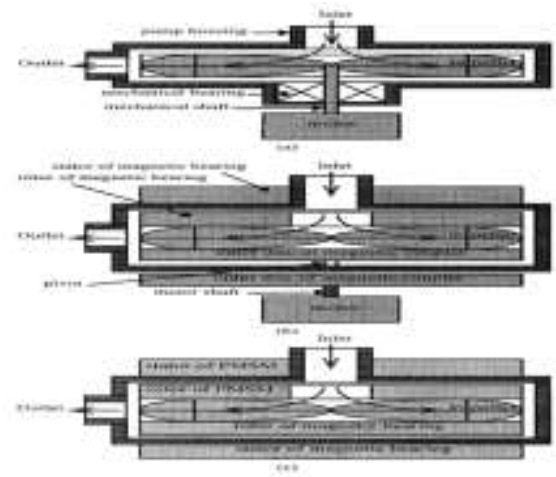

Figure 2. TAH with PMSM. (a) Motor coupled to impeller. (b) Motor coupled to magnetic couplers. (c) Magnetic bearing design.

\section{E. Brushless DC motor :}

The Streamliner pump was developed in 2002, brushless dc (BLDC) motor plays significant part in streamliner pump. This design is said to be third era design. It is very compact type design including with BLDC motor, mixed-flow impeller, and magnetic levitation components. To avoid the blood cell damage, the blood stream is slow enough and yet sufficiently quick to keep the development of clumps. Proper flow is accomplished by BLDC motor. Magnetic levitation type design was introduced to dispose of mechanical bearing wear.

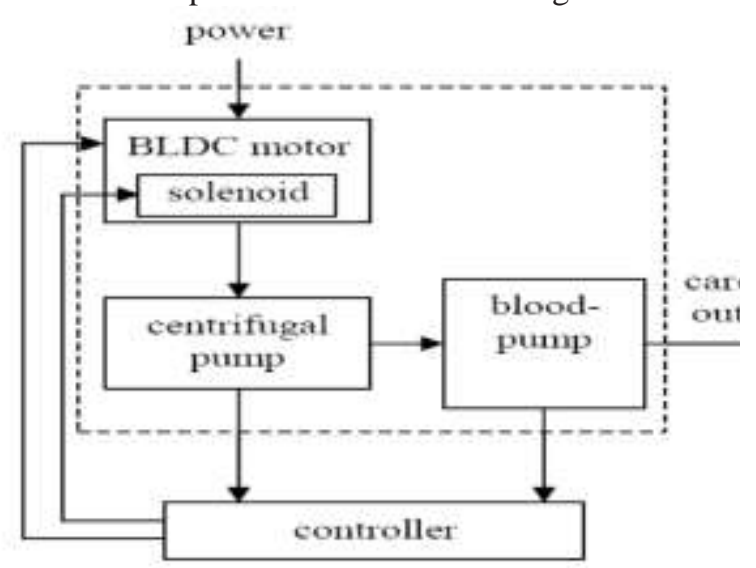

Figure.3. Block diagram of drive system.

Stream liner type design objective is to minimize the pump size, weight by rotating the pump impeller in the blood stream with magnetic levitation [5]. With a specific end goal to decrease the size and weight of the pump, change of the motor configuration is of incredible significance. In recent years BLDC motors are getting demand in industrial and medical applications due to their advantages like decreased size and weight, high effectiveness, enhanced dependability and great control qualities in a wide speed run [24].

\section{F. Axial self bearing motor :}

Axial self bearing motor is the improved development in artificial hearts. The fundamental highlights of the axial self-bearing motor in [25], has very little diameter under $30 \mathrm{~mm}$, and torque is more than $8 \mathrm{mNm}$ to enough pumping action. Axial self bearing motor with axial pump failed to achieve required flow. However, it is reported that flow rate of $2.8 \mathrm{l} / \mathrm{min}$ and pressure of $50 \mathrm{mmHg}$. Advanced improved development type design also given in[25] to increase flow rate or blood pumping capability. The advancement is that mixed flow, mixed flow is the combination of centrifugal and axial flow. Higher flow rates were achieved with association of mixed flow pump with axial- self bearing motor. Axial self-bearing motor was developed to work with small mixed flow pump with usage of magnetically suspended impeller. The axial self bearing motor drives the rotor up to $7600 \mathrm{r} / \mathrm{min}$ with levitating in air. double mixed flow pump with Magnetically-suspended motor was incorporated, results with this motor was recorded and reported flow rate of fluid at $5000 \mathrm{r} / \mathrm{min}$ is about $5.86 \mathrm{l} / \mathrm{min}$. However, the hemolysis test reported was unsatisfactory, hydrodynamic bearing was cause for hemolysis [25]. Some studies says that TAH is required to achieve a blood flow rate of $61 / \mathrm{min}$ [26]. Axial type self-bearing motor had high efficiency. Sufficient flow rate and pressure head is possible with axial self bearing motor.

\section{G. Other types :}

Other type design in artificial hearts and ventricular assist devices is that Bionic artificial heart blood pump. And it is driven by external permanent magnet, which is proposed in [28] to achieve pulsatility. This type blood pump design consists of active magnet and passive magnet. The dynamic magnet or active magnet is situated outside the body is driven by an external motor as per the direction of responding straight movement. Reciprocating linear motion starts with magnetic field generated by dynamic or active magnet situated outside the body. Under the impact of this attractive field, the passive magnet inside the body does likewise responding with linear motion according to active magnet, and brings the blood pump piston to do reciprocating linear motion. mechanical energy of motor is transferred to blood pump piston is done by active and passive magnets. Where blood pump piston is inside the human body, without any mechanical contact mechanical energy is transferred. And the purpose to provide power for the artificial heart blood pump is reached. Linear motors, small synchronus motors, small high speed DC motors are used to drive the active or dynamic magnet located outside the body in bionic type design [28].

Another different type of TAH and VAD is that operated with magnetic centrifugal pump, and external rotating magnetic field drives the associated pump. This design of Pump having a magnet rotor and which is synchronized to rotating magnetic 
field, so that fabricated impeller produces the required centrifugal force. Here pump is driven by the direct application of an external rotating magnetic field, this design eliminates the usage of shaft and impeller. Thus, the mechanism gives the advantages of small size, wireless operation, no requirement of battery, heat free operation and minimal risk of bacterial infections. length, Weight and diameter specifications are $35 \mathrm{~mm}, 34 \mathrm{~g}, 20 \mathrm{~mm}$, respectively, those specifications are specified in [27]. In recent era TAH, VADs, displacement pump designs are replaced with rotary pump devices. and it is reported that, it has other medical applications oriented practical advantages; which includes combined smaller weight and little size for better implantation, blood cell damage is very less compared to other type designs, smaller filling volume, and transportability also better. Requirements of implantable TAHs and VADs Implantable artificial heart systems are compact size, biocompatibility, and it should have higher durability. Particularly, most important thing is that compact size for implantable devices, it is especially for pediatric ventricular assist devices (VADs). Wireless operation and battery-free operation are additional advantages with this type design[28]. Several currently used devices are shown below:

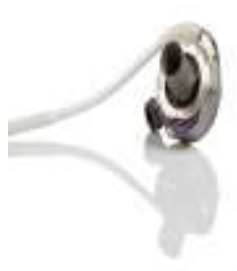

A

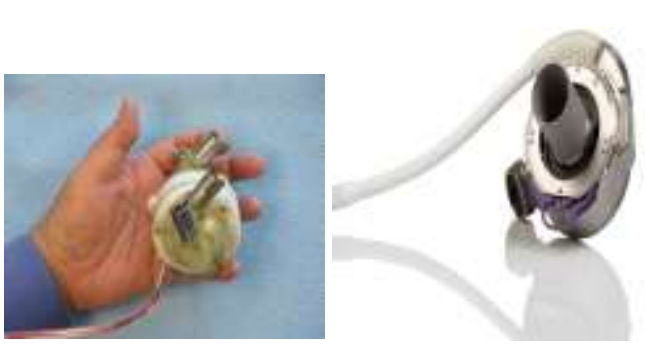

C

Figure. 2. (A) ThoratecHeartMates III leftventricularassistdevice, (B) Jarvik Infant device, (C) Continuous-flow totalartificial heart, (D) HeartMate 3

PUMPS USED IN ARTFICIAL HEARTS.

Pumps that are utilized as a part of VADs and TAHs are commonly separated into two classifications, pulsatile pumps and and continuous flow pumps. As the names recommend, pulsatile pumps mimics the beating idea of the body's cardiovascular framework, while continuous flow pumps produce a constant output flow from the device output. Reliability point of view continuous flow devices are best rather than pulsatile flow devices, because of reduced structure [17]. Researchers are focusing on Magnetic levitation and hydrodynamic bearings for use in centrifugal VADs and TAHs blood pumps, because of there significant advantages of reduced wear compared to intitial contact bearing systems. BiVAD (bi ventricular assist devices) and TAH support is still dominated by pulsatile devices, while Left-VAD development has moved toward third generation continuous flow devices which are continuous flow devices with pulsatile improvement techniques. Until the year 2010 there are no continuous flow single unit BiVADs on the market or involved in trials.

The essential distinction between centrifugal-flow and axial-flow pumps lies in the outline of their turning components. When one considers the hypothesis of operation of a centrifugal pump, its rotating element acts as a spinning disk with blades that can be viewed as a "thrower", implying that the liquid is caught and diverted extraneously out from the blade tips. Conversely, axial pump rotating components work like a propeller in a pipe and can be seen as a "pusher". This mechanism can likewise be seen as a "auger" ttempting to screw itself into the channel liquid, against the "resistance constrain" at the outlet, to defeat the distinction between preload and after-load [18]

\section{MECHANICAL WEAR AND IMPLANT LIFE.}

Continuous flow (CF) LVADs now being used as permanent destination treatment for end-stage heart failure patients, high reliability out to 5 years is generally considered a minimum requirement for permanent use, and a 10-year device is a targeted goal for newer device designs. Most centrifugal CF LVADs are defined as third-generation pumps in which the pump rotating element within the blood flow path is totally suspended during operation by some combination of hydrodynamic and/or magnetic (passive or active) forces, giving these pumps no life-limiting mechanical loads. Most axial (CF) pumps use second-generation rotating assemblies that are pivoted on small bearing surfaces in the blood flow path. An exception is the Berlin Heart INCOR axial CF LVAD, which uses magnetic bearings to actively suspend the rotating assembly. Bearing contact points in principle are pre-disposed to mechanical wear and heat generation, limiting the operating life of the pump. Despite the stated theoretic limits to pivoted bearing pumps, the Jarvik 2000 (Jarvik Heart Inc, New York, NY) and the HeartMate II axial rotary pumps 
have both shown excellent reliability, with no reported life-limiting wear of their blood immersed bearings. However, the German Heart Institute reported in 2010 that of 65 Berlin Heart INCOR and 18 MicroMed DeBakey axial rotary pumps implanted between 1991 and 2009, 3 stopped because of technical failure and 2 Berlin Heart INCOR devices had bearing problems. Interestingly, the HeartMate II axial pivoted-bearing CF pump was designed as a 5-year device, whereas the HeartMate III centrifugal CF pump, has a stated design goal of a 10 -year operating life.

The axial type pump is preferable for a centrifugal type, due to little structural design. So that, researchers and engineers introduced an axial pump with an axial self-bearing motor. Pumping of fluid with centrifugal force by centrifugal motor with the usage of flat rotors, radial blades. Whereas the axial type uses propellers for axial flow. Another type is reported that hybrid of the two types, that is mixed flow, which uses axially-expanded blades[25].

\section{Bearing design ;}

Bearings provide support to the rotating element (rotor). Following are several different kind of bearing elements:

Mechanical/pivo: Rotor is suspended with a mechanical arrangements such as mechanical bearings on spherical surfaces rotating in sockets (eg, Heart Mate II, Thoratec Corporation, Pleasanton, CA).

Hydrodynamic: Rotor gets lifting from fluid or liquid forces in thin, blood films isolating rotor and pump housing based on the relative movement of surfaces (eg, HeartWare HVAD, HeartWare International Inc, Framingham).

Electromagnet/position sensor: Rotor is suspended with electronic position control and electromagnets (eg, DuraHeart, Terumo Heart Inc, Ann Arbor, MI; and HeartMate III, Thoratec Corp).

Permanent magnet: Repelling magnets in rotor and pump housing suspend the rotor. Permanent magnets alone cannot suspend a rotating element because magnetic forces change continuously with the position of the rotor. Therefore, permanent magnets are generally used in combination with one of the other bearing types. The main difference in blood pump bearings lies in their complexity and reliability. Mechanical bearings have very small size and precise ceramic components whose advantage is that those are stable at all speeds and operating conditions, but the disadvantage of this type is that thrombus/fibrin deposition this is because of the bearing surface exposed to blood, which is not present in other bearing designs. Advantages of suspended type approach are that it provides the lowest shear stress to the blood because of relatively large clearances between the rotating element and the pump housing. However, these systems are complex, requiring position sensors, electromagnets and extra conductors, connector pins, and electronics to execute the dedicated position control algorithm. If, for example, there is an electrical contact failure in a connector pin, or momentary instability encountered in the control algorithm, pump failure can occur. To make the system failsafe, electromagnetic bearing elements are frequently backed up with hydrodynamic bearings, which require no control. Hydrodynamic bearings similarly produce no life limiting contact between the bearing surfaces, but in contrast, are simple and reliable. Once the rotor starts spinning, it essentially "water skis" on a film of blood, deriving lift and separation from its own motion. However, the load-bearing blood fluid film is prone to higher shear stress, and theoretically, more hemolysis can occur. Electromagnet bearing is more reliable compared to hydraulic type and mechanical type bearing [18]. Research is in progress reported in $[11,16,17]$ to improve not only the performance of electromagnet type bearing system, but also prevention of cell damage.

\section{Technical challenges in VADs \& TAHs.}

Before starting a design of VADs or TAH, The design engineers have to look following requirements.

- Because of the restricted space in the human thorax, the measurements of the TAH ought not to surpass $85 \mathrm{~mm}$ in breadth and $95 \mathrm{~mm}$ long.

- The aggregate weight of the TAH ought to be under $800 \mathrm{~g}$, contrasted with $400 \mathrm{~g}$ of the normal heart. So size of Electrical motor and mechanical pump ought to be less.

- The normal pumping limit should add up to $6 \mathrm{l} / \mathrm{min}$ against a medium aortic weight of $100 \mathrm{mmHg}$ and provides an extra over-burden limit.

- It is reported that, The electrical losses must be lesser than $20 \mathrm{~W}$. if it is more than $20 \mathrm{~W}$, it leads to blood damage due to heat tolerance of the human body.

- To pump the blood properly, The Electrical motor and pump has to provide a maximum force of $70 \mathrm{~N}$. - A long durability (5 years) of the TAH by a wear free and/or redundant system is desirable. Above all points are technical challenges for engineers to meet the activities of natural heart, those are reported in [31].

\section{Conclusion:}

Continuous flow VADs (CFVADs) are smaller due to avoiding of larger mechanical elements, and also CFVADs are more durable, reliable than pulsatile flow VADs (PFVADs) [29]. However, CFVADs produce continuous-flow $(\mathrm{CF})$ and diminished 
pulsatility systemically. pulsatile flow is the basic nature of human heart. In view of these perceptions, there is at present an enthusiasm for creating control techniques for CFVADs as a method to generate a pulse pressure (PP) [11]. HeartMate 3 (St. Jude Medical Inc.) is a smaller profile, fully magnetically levitated centrifugal pump that is undergoing an IDE trial in the USA (Momentum 3 IDE Trial) and is already approved for clinical use in European Union. The pump incorporates artificial pulse technology, which may reduce some of the complications that are attributed to lack of adequate pulse pressure in patients supported with current centrifugal pumps [17]. And it is required to improve the performance of electromagnet bearing system.

Newer devices should have to overcome the current disadvantages of the TAHs and VADs which are:

1. Bulky and may not fit in many patients in need.

2. Patients are tethered to the driver (Cardiowest TAH) which interferes with quality of life.

3. If considered for destination therapy, durability is not illustrated.

4. Thromboembolism, infections are secondary complications.

5.Quiet inconvenience from sound-related unsettling influences.

6. Lack of pressure flow relationship that mimics native heart to adjust for varying loading conditions. [30]

The motor which is used in MCSD should be able to achieve pulsatile flow or continues flow. Motor should be very small size and it have to provide required force to pump the blood. Most motors and pump assemblies reported to achieve the pulsatality are not compact as they require externally placed drive. Implantable drives would lead to infection. Compact motor and pump assemblies without the need for external drive would help a long way in decreaseing the patient's mortality rate because of Heart failure.

\section{References}

1. B. A. P, Felix Lamping, Gonzalo Alvarez, Marco Laumen, P. Carstens, Thomas Finocchiaro, Ulrich Steinseifer and Y. M. Blaszczyk Novel Optical Position Sensing for Miniaturized Applications and Validation in a Total Artificial Heart. IEEE transaction on Biomedical engineering, Vol 63, No 3 3, March2016.

2. Axel Haverich, CircuLite Inc, Daniel Burkhoff, Gwen Buechler, Jan D. Schmitto, Martin Strueber, Murat Avsar, New Jersey, Oliver Fey, Petra Ziehme, and Saddle Brook. Two axial-flow Synergy Micro-Pumps as a biventricular assist device in an ovine animal model; The Journal of Heart and Lung Transplantation, Vol 31, No 11, November 2012.
3. Leslie mertz From artificial kidneys to artificial heart and beyond. IEEE Pulse May/June 2012.

4. Sean P. Pinney. Left Ventricular Assist Devices: The Adolescence of a Disruptive Technology. Journal of Cardiac Failure Vol. 21 No. 102015.

5. By James Antaki, Brad E. Paden, Michael J. Piovoso, and Siva S. Banda "Award Winning Control Applications"; IEEE Control Systems Magazine December 2002.

6. Chad E. Eckert, Daniel J. Goldstein, David C. Naftel, Robert L. Kormos, William L. Holmanand James K. Kirklin Durability of left ventricular assist devices: Interagency Registry for Mechanically Assisted Circulatory Support (Intermacs). The Journal of Thoracic and Cardiovascular Surgery. August 2013.

7. Salim Yusuf, Sonia Anand, Srinath Reddy and Stephanie Ounpuu. Global Burden of Cardiovascular Diseases Part I: General Considerations, the Epidemiologic Transition, Risk Factors, and Impact of Urbanization. Pgs 2746-2753 November 27, 2001 doi.org/10.1161/hc4601.099487.

8. Francis D. Pagani "The quest toward the Holy Grail of mechanical circulatory support"; The Journal of Thoracic and Cardiovascular Surgery,September2015.

9. Eric Maslen, Paul Allaire, RonaldFlack, and Edgar Hilton, Michael Baloh, Don Olsen, Gill Bearnson, and James D.Long "feed back control applications in artifiial hearts" ; 0272- 1708/98/\$10.0001998 IEEE Control Systems. December 1998.

10. James K. Kirklin, David C. Naftel, Francis D. Pagani, Robert L. Kormos, MD, Lynne Stevenson, Marissa Miller, DVM, MPH, and James B. Young, MD "Long-term mechanical circulatory support (destination therapy): On track to compete with heart transplantation?"; The Journal of Thoracic and Cardiovascular Surgery c September 2012.

11. Allen Cheng, Christine A. Williamitis, and Mark S. Slaughter A journel "Comparison of continuous-flow and pulsatile-flow left ventricular assist devices", November 2013.

12. Marcus Granegger, Marco Masetti, Ravi Laohasurayodhin, Thomas Schloeglhofer, Daniel Zimpfer, Heinrich Schima, and Francesco Moscato "Continuous Monitoring of Aortic Valve Opening in Rotary Blood Pump Patients" ; IEEE Transactions on Biomedical engineering, Vol. 63, no. 6, June 2016. 
13. Jinghua Ji, Jianxing Zhao, Wenxiang Zhao, Member, IEEE, Zhuoya Fang, Guohai Liu, Member, IEEE, and Yi Du "New High Force Density Tubular Permanent-Magnet Motor" ; IEEE Transactions on Applied Super Conductivity, VOL. 24, NO. 3, JUNE 2014.

14. Selim Bozkurt, Sjoerdvan Tuijl, Stephanie Schampaert, Frans N. van de Vosse,Marcel C.M. Rutten on "Arterial pulsatility improvement in a feedback-controlled continuousflow left ventricular assist device: An ex-vivo experimental study" ; 2014 IPEM. Published by Elsevier Ltd.

15. Nicholas A. Greatrex, Daniel L. Timms, Nobuyuki Kurita, Edward W. Palmer, and Toru Masuzawa on "Axial Magnetic Bearing Development for the Bivacor rotary BiVAD/TAH" ; IEEE Transactions on Biomedical engineering, vol. 57, no. 3, march 2010.

16. Jamshid H. Karimov, Nader Moazami, Mariko Kobayashi, Shiva Sale, Kimberly Such, Nicole Byram, Gengo Sunagawa, David Horvath, Shengqiang Gao, Barry Kuban, Leonard A. R. Golding, Fracs, and Kiyotaka Fukamachi. "First report of 90day support of 2 calves with a continuousflow total artificial heart" ; The Journal of Thoracic and Cardiovascular Surgery , September 2015.

17. Deshpande, K.O. Maher, D.L. Morales on "Mechanical Circulatory Support in Children: Challenges and Opportunities", August2016.

18. Kiyotaka Fukamachi, Akira Shiose, Alex L. Massiello, David J. Horvath, Leonard A. R. Golding, Fracs, Sangjin Lee, MD, and Randall C. Starling, "Implantable Continuous-Flow Right Ventricular Assist Device: Lessons Learned in the Development of a Cleveland Clinic Device"; 2012 by The Society of Thoracic Surgeons Published by Elsevier Inc.

19. Thomas Finocchiaro, Thomas Butschen, Paul Kwant, Ulrich Steinseifer, Thomas Schmitz-Rode, Kay Hameyer, and Marc Lebmann on "New Linear Motor Concepts for Artificial Hearts"; IEEE Transactions on Magnetics, VOL. 44, NO. 6, JUNE 2008.

20. Jinghua Ji, Wenxiang Zhao, Zhengmeng Liu, and Qian Chen on "A Novel DoubleStator Tubular Vernier Permanent-Magnet Motor With High Thrust Density and Low Cogging Force";IEEE Transactions On Magnetics, Vol. 51, No. 7, July 2015.

21. Jinghua Ji1, Zhijian Ling1, Jiabin Wang, Wenxiang Zhao1, Guohai Liu1, and Tao Zeng "Design and Analysis of a Halbach Magnetized Magnetic Screw for Artificial
Heart"; IEEE Transactions On Magnetics, Vol. 51, No. 11, November 2015.

22. Jinghua Ji, Shujun Yan, Wenxiang Zhao, Guohai Liu, and Xiaoyong Zhu on "Minimization of Cogging Force in a Novel Linear Permanent-Magnet Motor for Artificial Hearts"; IEEE Transactions On Magnetics, Vol. 49, No. 7, July 2013..

23. J. X. Shen, Member, IEEE, K. J. Tseng, Senior Member, IEEE, D. M. Vilathgamuwa, Senior Member, IEEE, and W. K. Chan on "A Novel Compact PMSM with Magnetic Bearing for Artificial Heart Application"; IEEE Transactions On Industry Applications, VOL. 36, NO. 4, July/August 2000.

24. ABDEL-KARIM DAUD on "Two Phase Brushless D.C. Motor For Artificial Heart Applications"; International Journal of Biology and Biomedical Engineering Volume-3 2009.

25. Yohji Okada, Naoto Yamashiro, Kunihiro Ohmori, Toru Masuzawa, Member, IEEE, Takashi Yamane, Yoshiaki Konishi, and Satoshi Ueno, Member, IEEE "Mixed Flow Artificial Heart Pump With Axial Self-Bearing Motor"; IEEE/ASME Transactions on Mechotronics, VOL. 10, NO. 6, December 2005.

26. André Pohlmann, Marc Leßmann, Thomas Finocchiaro, Thomas Schmitz-Rode, and Kay Hameyer "Numerical Computation Can Save Life: FEM Simulations for the Development of Artificial Hearts"; IEEE Transactions on Magnetics, VOL. 47, NO. 5, May 2011.

27. S. H. Kim, S. Hashi, and K. Ishiyama on "Actuation of Novel Blood Pump by Direct Application of Rotating Magnetic Field";IEEE Transactions on Magnetics, VOL. 48, NO. 5, May 2012.

28. Dong Xia ; "A Bionic Artificial Heart Blood Pump Driven by Permanent Magnet Located Outside Human Body"; IEEE Transactions on Applied Superconductivity, Vol. 22, No. 3, June 2012.

29. Nadermoazami,KiyotakaFukamachi,Marik oKobayashi,NicholasG.Smedira,

KatherineJ.Hoercher, AlexMassiello, Sangjinlee, David J.Horvath, Randall C. Starling, "Axial and centrifugal continuous-flow rotary pumps: A translation from pump mechanics to clinical practice";2013 International Society for Heart and Lung Transplantation.

30. Shiva M. Sale, Anesthesiologist, Nicholas G. Smedira, Surgical Director "Total 
artificial heart"; Best Practice \& Research Clinical Anaesthesiology 262012

31. André Pohlmann, Marc Leßmann, Thomas

Finocchiaro, Thomas Schmitz-Rode, and

Kay Hameyer on "Numerical Computation

Can Save Life: FEM Simulations for the

Development of Artificial Hearts"; IEEE

Transactions on Magnetics, Vol. 47, NO. 5,

May2011 